\title{
Factors influencing electrochemically enhanced sacrificial anode phosphate recovery
}

\author{
Xiaoyan $\mathrm{ZHOU}^{1}$, and Gang $\mathrm{ZHANG}^{1 *}$, Haijian $\mathrm{SHI}^{2}$ \\ ${ }^{1}$ School of Civil Engineering and Architecture, University of Jinan, 336 Nanxinzhuang West Road, Jinan, 250022, China \\ ${ }^{2}$ Weihai ecological environment bureau wendeng branch,95 Fengshan Road Wendeng District, Weihai, 264400, China
}

\begin{abstract}
The recovery of phosphate from wastewater has been attracting increasing attention. In this study, the technology of an electrochemically enhanced sacrificial anode was applied to investigate the phosphate recovery process from sewage. The experimental results indicated that, when residence times ranged from 20 to $120 \mathrm{~s}$, and phosphate removal efficiencies ranged from $24.65 \%$ to $95.27 \%$. When the current density ranged from 10 to $70 \mathrm{~A} / \mathrm{m}^{2}$, the phosphate removal efficiencies ranged from $56.73 \%$ to $95.17 \%$. When the plate spacing ranged from 1 to $5 \mathrm{~cm}$, the phosphate removal efficiencies ranged from $83.48 \%$ to $9.48 \%$. The experimental results confirm the conclusions of this study about controlling operational factors in practical applications to increase phosphate removal efficiencies.
\end{abstract}

\section{Introduction}

As we all know, phosphate resources are gradually decreasing on the earth ${ }^{[1]}$. To effectively recover phosphate resources, several techniques of recycling phosphate (e.g., adsorption and precipitation) have been explored ${ }^{[2]}$. Phosphate can be removed by using coagulants and adsorbents ${ }^{[3]}$. Iron salts and aluminum salts are commonly used coagulants for the removal of phosphate; these include ferrous sulfate, ferric chloride, and polyaluminum chloride. Adsorption has the advantage of fast adsorption speed and simple operation, but there are problems such as dissolution loss of adsorbent and difficulties in regeneration. Precipitation not only entails adding chemical agents but uses chemical reactions to generate precipitation and thus removes the phosphate in the wastewater. The work of Kruk et $\mathrm{a}^{[4]}$ shows that struvite precipitation using a magnesium sacrificial anode for phosphate recovery is technically feasible. They find that, when high-purity magnesium alloy is used as an anode, not only the removal of phosphate but the recovery of struvite can be highly efficient in the wastewater treatment of a supernatant of fermented waste activated sludge from wastewater. Precipitation has the advantages of simple operation and strong impact load resistance, but the operational cost is high. Some researchers have been concerned with the electrochemical reduction of nitrates by using different cathodes such as $\mathrm{Cu}, \mathrm{Ti}, \mathrm{Ru}$, and $\mathrm{Fe}^{[5]}$. In the electrochemical process, the cathode can produce hydrogen, which is an electron donor, from nutrient denitrifying bacteria. Iron cathodes have been shown to be relatively effective in promoting nitrate reduction ${ }^{[6]}$. On this basis, the electrochemical phosphate removal process can be added, and it can treat not only nitrates but also phosphates ${ }^{[7]}$. The electrochemical phosphate removal process has been used on domestic sewage and it now finds increased application in a range of small- and medium-sized companies, and it also has been received more and more attention ${ }^{[8]}$.

Therefore, in the current study, the phosphate removal effect of different operating conditions, such as plate spacings, current densities, and residence time were studied by using an electrochemically phosphate recovery process.

\section{Materials and methods}

\subsection{Materials}

The secondary treatment water in a community water station was used as the treatment object. The raw water of the reclaimed water station was domestic sewage, and we adopted aeration regulation + hydrolysis acidification + anoxic treatment + biochemical treatment + precipitation + filtration + disinfection as the main treatment process. The typical sewage has a $\mathrm{pH}$ of $7.0-8.5$ and a phosphate content of 3-5 mg/L.

\subsection{Experimental system}

As shown in Fig. 1, the electrochemical phosphate removal reaction takes place in a rectangular container. The rectangeular container was made of plexigalss. The dimensions of the electrode plates used in these experiments $(\mathrm{Fe})$ were $10 \times 100 \times 100 \mathrm{~mm}$. The water temperature and voltage were measured during all the experiments at a sampling interval of 5-40 min. The $\mathrm{pH}$ values of the solution were measured before and after each experiment. All experiments were at least duplicated, and 
the results showed good repeatability.

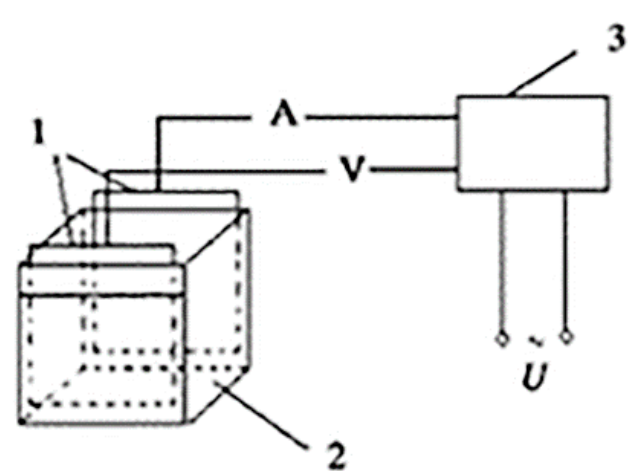

1: electrode plate material

2: electrochemical reactor

3: DC power

Fig1. Laboratory-scale electrochemical system used in the experiments.

\subsection{Analytical method}

All glass materials that came in contact with the water samples were rinsed with $\mathrm{HCl}$ acid and pure water in advance. Water samples were filtered through a $\varphi 15 \mathrm{~cm}$ filter paper. Phosphate removal was measured using the UV spectrophotometer UV-5800 (Yuanxi, China). The $\mathrm{pH}$ value was determined with a $\mathrm{pH}$ meter (FE20K, Mettler Toledo, USA). The control of the current was supplied by DC power (DPS-3005D, Zhaoxin, China).

\section{Results and discussion}

\subsection{Effect of residence time}

Residence time has an important effect on the electrochemically enhanced sacrificial anode phosphate recovery process, because the parameter is directly dependent on the dissolved anode metal. Therefore, the effect of residence time on the phosphate removal efficiency was studied within the range of 20-120 s at a current density of $10 \mathrm{~A} / \mathrm{m}^{2}(i=0.1 \mathrm{~A})$, with a plate spacing of $2 \mathrm{~cm}$, an iron anode, and an initial neutral $\mathrm{pH}$ condition. Fig. 2 shows the relationship between phosphate removal efficiency and residence time.

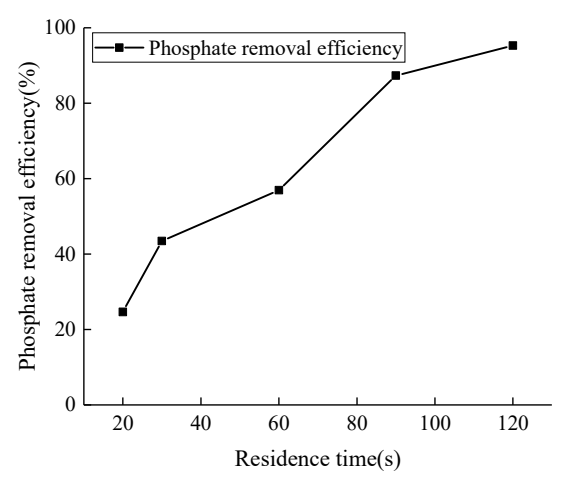

Fig2. Effect of residence time on phosphate removal efficiency.

As observed from Fig. 2, longer residence time increased the effect on the efficiency of phosphate removal in the electrochemically enhanced sacrificial anode phosphate recovery process. When the residence time was from 20 to $60 \mathrm{~s}$, the rate of phosphate removal increased from $24.65 \%$ to $56.93 \%$. In this residence time interval, the phosphate removal rate increased rapidly with the increase of residence time. When the residence time increased from 60 to $120 \mathrm{~s}$, the rate of phosphate removal increased from $56.93 \%$ to $95.27 \%$. In this residence time interval, the rate of phosphate removal increases with the increase of residence time gradually slowed. To save on power cost, in subsequent experiments a residence time of $60 \mathrm{~s}$ was used.

Because of the constant current density, the amounts of ferrous ions and iron ions both gradually increased with the extension of residence time, and the precipitation of ferrous phosphate and iron phosphate was greatly increased by ferrous ions and iron ions combining with phosphate. Ferrous ions and iron ions would hydrolyze to form flocculants such as ferric hydroxide and ferrous hydroxide, which could adsorb phosphate. Hence, the removal rate of phosphate increased with the residence time. However, the anode was likely to become passivated as the residence time increased. A dense oxide film was formed on the surface of the iron electrode that inhibited the anode from continuing to dissolve and release ferrous ions and iron ions. Consequently, the residence time has little effect on the phosphate removal efficiencies.

\subsection{Effect of plate spacing}

Plate spacing is one of the operational parameters that greatly influences the removal efficiencies of phosphate from reclaimed water using the electrochemically enhanced sacrificial anode phosphate recovery process. The effect of plate spacing on the removal of phosphate was explored within the range of $1-5 \mathrm{~cm}$ at a current density of $10 \mathrm{~A} / \mathrm{m}^{2}(i=0.1 \mathrm{~A})$, with an initial neutral $\mathrm{pH}$ condition, using an iron anode, and with a residence time of $1 \mathrm{~min}$. The optimum plate spacing and corresponding phosphate removal percentage in this study are presented in Fig. 3. 


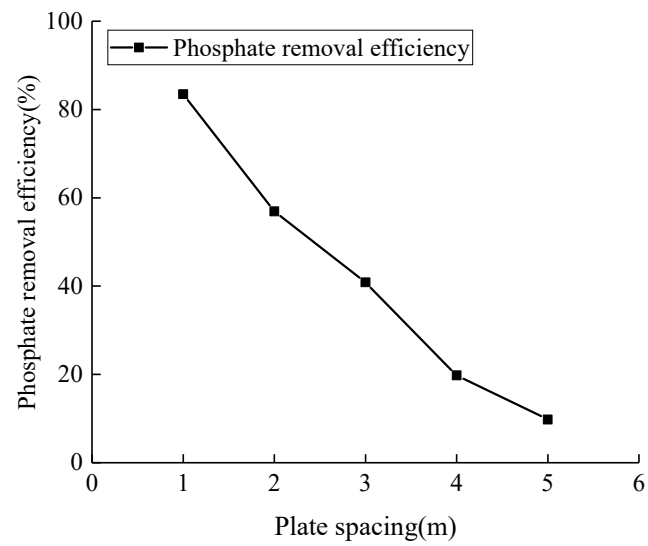

Fig3. Effect of plate spacing on phosphate removal efficiency.

Fig. 3 shows that plate spacing can affect the phosphate removal efficiency. When the plate spacing was increased from 1 to $5 \mathrm{~cm}$, the phosphate removal efficiency decreased from $83.48 \%$ to $9.48 \%$. Therefore, the longer the plate spacing, the more the phosphate removal rate would gradually decrease.

As a result of the smaller plate spacing, the current density flowing between the plates was higher, which produced more ferrous ions and iron ions ${ }^{[9]}$.Ferrous ions and iron ions could combine with more phosphate to precipitate ferrous phosphate and iron phosphate. Based on this study, because the plate spacing affected the phosphate removal efficiencies, it is necessary to choose a suitable plate spacing for the electrochemically enhanced sacrificial anode phosphate recovery process. Given the influence of actual processing and other factors, a plate spacing of $2 \mathrm{~cm}$ was chosen in subsequent experiments.

\subsection{Effect of current density}

Current density is a crucial parameter for controlling the reaction rate in the electrochemically enhanced sacrificial anode phosphate recovery process. The operational current density is critical to the electrochemically enhanced sacrificial anode phosphate recovery process as it is the only operational factor that can be controlled directly ${ }^{[10]}$. The effect of current density on the removal of phosphate was explored within the range of $10-70 \mathrm{~A} / \mathrm{m}^{2}$ at a plate spacing of $2 \mathrm{~cm}$, with an initial neutral $\mathrm{pH}$ condition, using an iron anode, and with a residence time of 1 min. Fig. 4 demonstrates the effect of current density on the phosphate removal percentage.

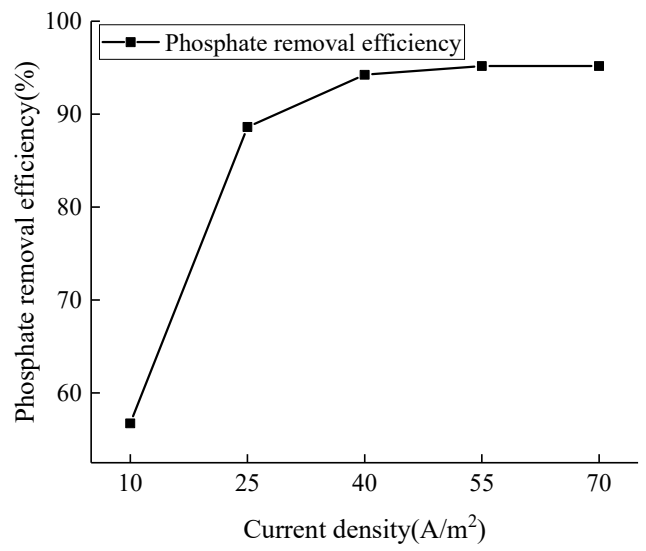

Fig4. Effect of current density on phosphate removal efficiency.

As can be seen in Fig. 4, the results indicate that current density can make a difference in the phosphate removal efficiency. When the current density increased from $10 \mathrm{~A} / \mathrm{m}^{2}$ to $40 \mathrm{~A} / \mathrm{m}^{2}$, the phosphate removal efficiency increased from $56.73 \%$ to $94.23 \%$ and the phosphate removal rate was faster. When the current density increased from $40 \mathrm{~A} / \mathrm{m}^{2}$ to $70 \mathrm{~A} / \mathrm{m}^{2}$, the phosphate removal efficiency increased from $94.23 \%$ to $95.17 \%$ and the phosphate removal rate slowed down. Thus, the optimal current density is $40 \mathrm{~A} / \mathrm{m}^{2}$.

On the basis of Faraday's law, the current density can attribute to the increase in dissolution rate of the sacrificial iron ele 0 ctrode $^{[11]}$. The amounts of dissolved ferrous ions and iron ions were greatly increased. As the current density increased, the amounts of ferrous ions and iron ions increased in the anode plate per unit time, and the chance of ferrous ions and iron ions colliding with phosphate to form precipitates of ferrous phosphate and iron phosphate was greatly increased. In addition, the increase of ferrous ions and iron ions also enhanced the flocculation of the electrochemical reaction, and many bubbles were produced during the high-current-density electrochemical reaction, so that these effects were both beneficial for high phosphate removal efficiencies ${ }^{[12]}$.

\section{Conclusion}

The phosphate removal effects of residence time, plate spacing, and current density by using a process of electrochemically phosphate recovery have been explored. A range of electrochemically phosphate recovery process options were thoroughly investigated to assess the performance and maintenance requirements of various actual scenarios. Under the same experimental conditions, our results reveals that the optimum operating conditions are plate spacing is $2 \mathrm{~cm}$, residence time of $60 \mathrm{~s}$ and current density of $40 \mathrm{~A} / \mathrm{m}^{2}$ should be considered. During operation, the phosphate removal percentage was found to increase with both longer residence time and higher current density. Therefore, the electrochemical process ensures that the process is very efficient, reduces overall energy, and shortens retention time. However, in practice, the 
optimum operating conditions need to be a harmonious combination among these factors for the greatest efficiency and cost savings.

\section{Acknowledgment}

This study was financially supported by the Natural Science Foundation of Shandong Province (ZR2017MEE061) and the Key Research and Development Project of Shandong Province (2016GSF117007). The authors would like to thank reviewers and the manuscript editors for their constructive comments.

\section{Author Disclosure Statement}

No competing financial interests exist.

\section{References}

1. L.L.E Bayon. F.C. Ballesteros Jr., S.G Segura, M.C $\mathrm{Lu}$, Water reuse nexus with resource recovery: On the fluidized-bed homogeneous crystallization of copper and phosphate from semiconductor wastewater. J. Clean. Prod, 236, 117705 (2019)

2. J.S Wei, X.G Meng, X.H Wen, Y.H Song, Adsorption and recovery of phosphate from water by amine fiber, effects of co-existing ions and column filtration. J Environ Sci-China, 87, 123 (2020).

3. Y.B Chu, M Li, J.W Liu, W Xu, S.H Cheng, Molecular insights into the mechanism and the efficiency-structure relationship of phosphorus removal by coagulation. Sci. Water Research, 147, 195 (2018).

4. D.J Kruk, M Elektorowicz, J.A Oleszkiewicz, Struvite precipitation and phosphorus removal using magnesium sacrificial anode. Sci. Chemosphere, 101, 28 (2014).

5. Y Gao, Y.W Xie, Q Zhang, Y.X Wang, L.Y Yang, Intensified nitrate and phosphorus removal in an electrolysis-integrated horizontal subsurface flow constructed wetland. Sci. Water Research, 108, 39 (2017).

6. X.X Ju, S.B Wu, Y.S Zhang, R.J Dong, Intensified nitrogen and phosphorus removal in a novel electrolysis-integrated tidal flow constructed wetland system. Sci. Water Research, 108, 37 (2017).

7. D.D Nguyen, H.H Ngo, W Guo, T.T Nguyen, S.W Chang, A Jang, Y.S Yoon, Can electrocoagulation process be an appropriate technology for phosphorus removal from municipal wastewater. Sci. Sci Total Environ, 563, 549 (2016).

8. V Kuokkanen, T Kuokkanen, J Ramo, U Lassi, J Roininen, Removal of phosphate from wastewaters for further utilization using electrocoagulation with hybrid electrodes-Techno-economic studies. Journal of Water Process Engineering, 8, 50 (2015).

9. A Shalaby, E Nassef, A Mubark, M Hussein, Phosphate removal from wastewater by electrocoagulation using aluminium electrodes. Sci. Environ Eng Sci, 1, 90 (2014).
10. X.Y Zheng, H.N Kong, D.Y Wu, C Wang, Y Li, H.R Ye, Phosphate removal from source separated urine by electrocoagulation using iron plate electrodes. Water Sci Technol, 60, 2929 (2009).

11. A Attour., G.N Ben, T.M Mouldi, F Lapicque, J.P Leclerc, Intensification of phosphate removal using electrocoagulation treatment by continuous $\mathrm{pH}$ adjustment and optimal electrode connection mode. Sci. Desalin Water Treat. 57,13255 (2016). 